\title{
FLUX-CORED WIRES FOR SURFACING OF STEEL HOT MILL ROLLS
}

\author{
I.A. KONDRATIEV and I.A. RYABTSEV \\ E.O. Paton Electric Welding Institute, NASU \\ 11 Bozhenko Str., 03680, Kiev, Ukraine. E-mail: office@paton.kiev.ua
}

\begin{abstract}
The flux-cored wires and technologies of arc surfacing of steel hot mill rolls were developed. The results of investigations and practical experience allows recommending the developed flux-cored wires for surfacing of steel rolls of the following mills: for roughing (blooming and slab) - PP-Np-25Kh5MSGF; for continuous billet - PP-AN147, PP-Np-35V9Kh3GSF; for heavy-section and rail-beam - PP-Np-25Kh5MSGF; for medium- and light-section - PP-Np-25Kh5MSGF, PP-AN147, PP-AN204; for wire - PP-Np35V9Kh3GSF, PP-AN132; for sheet - PP-AN132, PP-Np-25Kh5MSGF; for pipe ones - PP-AN147, PP-Np-35V9Kh3GSF. 3 Ref., 1 Table, 1 Figure.
\end{abstract}

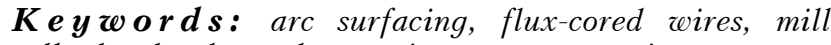
rolls, hot hardness, heat resistance, wear resistance

Nowadays the surfacing of mill rolls for their restoration and increase in life is applied almost at all the metallurgical enterprises in Ukraine. Using modern methods of mechanized surfacing a roll can be created with a very ductile and strong core, which is well resistant to mechanical loads and also has a wear- and heat-resistant surface. Surfacing allows significantly increasing of life of rolls, decreasing their consumption, increasing yield of efficient rolled metal due to improvement of accuracy of rolling, decreasing expenses for processing and cost of rolled metal [1].

The efficiency of application of surfacing of mill rolls depends significantly on right selection of composition of deposited metal. Therefore, it is necessary to perform a thorough analysis of conditions of rolls operation, character and intensity of their wear. At different metallurgical enterprises the rolls of mills even of the same type are worn out to different extent and should be surfaced using different consumables.
For wear-resistant surfacing of steel hot rolls of different mills most often, though not always reasonably, the flux-cored wire of grade PP-Np35V9Kh3GSF is applied. The deposited metal of $\mathrm{Cr}-\mathrm{W}$ type of steel possesses a high resistance to abrasion at higher temperatures but its thermal life is comparatively low, and the rolls, deposited using this wire, often come out of order due to formation of fire net and spallings. Therefore, to deposit the rolls using this wire, to which the requirements of maximum cleanness of surface of body or grooves of a roll are specified, is not rational.

The experience in development of steels for dies of hot deformation of metals, conditions of work of which are largely close to those for work of hot mill rolls, indicates the challenge in use of $\mathrm{Cr}-\mathrm{W}-\mathrm{Mo}$ (partial replacement of tungsten with molybdenum) and $\mathrm{Cr}-\mathrm{Mo}$ steels for these purposes. As to heat resistance, these steels are almost not inferior to $\mathrm{Cr}-\mathrm{W}$ ones and, as to resistance to thermal fatigue, are significantly superior to them. It is connected with the fact that molybdenum facilitates formation of fine-grain

Properties of deposited metal of different alloying systems

\begin{tabular}{|l|c|c|c|c|c||}
\hline \multicolumn{1}{|c|}{ Grade of flux-cored wire } & $\begin{array}{c}\text { Heat resistance, } \\
\text { number of cycles }\end{array}$ & $\begin{array}{c}\text { Wear of specimen } \\
\Delta M, \mathrm{~g}\end{array}$ & Heat resistance $T_{\mathrm{d}},{ }^{\circ} \mathrm{C}$ & $\begin{array}{c}\text { Impact toughness } a_{\mathrm{n}}, \\
\mathrm{J} / \mathrm{cm}^{2}\end{array}$ & Hardness $H R C$ \\
\hline PP-Np-35V9Kh3GSF & 70 & 0.12 & 680 & 7 & 51 \\
\hline PP-Np-25Kh5MSGF & 200 & 0.35 & 650 & 35 & 46 \\
\hline PP-AN147 & 190 & 0.15 & 650 & 67 & 50 \\
\hline PP-AN132 & 130 & 0.13 & 650 & 23 & $29-50^{*}$ \\
\hline PP-AN204 & 170 & 0.21 & & \\
\hline${ }^{*}$ After ageing at $480{ }^{\circ} \mathrm{C}$ for $3 \mathrm{~h}$.
\end{tabular}




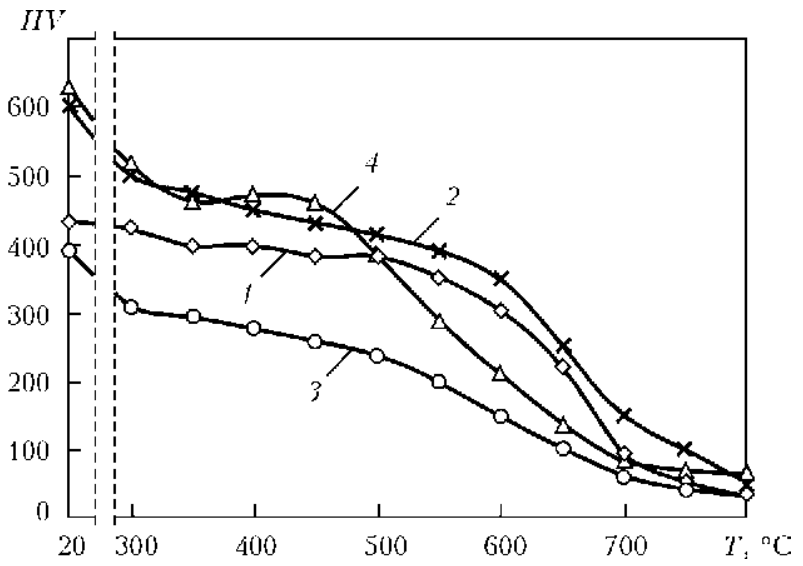

Hot hardness of deposited metal: 1 - surfacing with wire PP-Np-25Kh5MSGF; 2 - PP-Np-35V9Kh3GSF; 3 - steel $150 \mathrm{KhNM}$ hardened and tempered for hardness $H R C 50$ 4 - PP-AN204

structure, hinders precipitation of carbide particles along the grain boundaries and, thus, increases the ductility of steel.

For surfacing of layer of $\mathrm{Cr}-\mathrm{Mo}$ steel the fluxcored wire of grades PP-Np-25Kh5MSGF and PP-AN147, and for Cr-W-Mo steel PP-AN132 one were developed. The properties of metal deposited by these wires are presented in the Table.

The heat resistance was determined by a number of heating-cooling cycles until the appearance of crack net became visible by a naked eye. The wear resistance was evaluated by the loss of mass $\Delta M$ of deposited specimen caused by friction wear of metal against the metal at $600{ }^{\circ} \mathrm{C}$ for $1 \mathrm{~h}$ of tests. The heat resistance of deposited metal $T_{\mathrm{d}}$ was characterized by the temperature of twohour tempering, after which the hardness amounted to $H R C 40$.

Tungsten-free metal, deposited using wires PP-Np-25Kh5MSGF and PP-AN147, has the highest resistance to thermal fatigue and the best combination of values of heat and wear resistance belongs to the metal, deposited using wire PPAN147.

During surfacing of rolls with complicated grooves the mechanical treatment of deposited layer encounters great difficulties due to relatively high hardness. For such rolls the application of surfacing materials of the type of maraging or dispersion-hardening steels and, in the first turn, sparcely alloyed and tool maraging steels is challenging. A high strength of steels of the mentioned group is a total result of realization of mainly two hardening processes: formation of interstitial solid solution and shear (martensite) mechanism of $\gamma-\alpha$ transformation. After surfacing such steels have hardness of HRC 28-30 and are sufficiently easily treated mechanically. After tempering the hardness is increased to $H R C$ 4855 , and the deposited metal acquires high service properties. Besides, it enables carrying out of surfacing without preliminary and concurrent preheating.

To deposit a layer of maraging steel of alloying $\mathrm{Fe}-\mathrm{Ni}-\mathrm{Mn}-\mathrm{Si}-\mathrm{Mo}$ system the flux-cored wire PP-AN204 was developed [2, 3]. The main properties of metal deposited using this wire are presented in the Table. Besides, hot hardness of deposited metal as compared to the deposited metal of the type of known tool steels was investigated (Figure).

Heating of specimens was performed in the special inductor in vacuum, measurements of hardness were carried out at $1 \mathrm{~kg}$ loading and $60 \mathrm{~s}$ holding. It is seen from the given data that hot hardness of maraging deposited metal is at the same level as hot hardness of $\mathrm{Cr}-\mathrm{Mo}$ and $\mathrm{Cr}-\mathrm{W}$ die steels, deposited with the corresponding flux-cored wires.

According to the results of laboratory investigations and pilot-industrial verifications, performed in the recent years, the compositions of deposited metal and, respectively, compositions of charge of flux-cored wires for surfacing of hot mill rolls were specified. The results of investigations and practical experience allow recommending any of the developed flux-cored wires for surfacing of steel rolls of the following mills: for roughing (blooming, slab) - $\mathrm{PP}-\mathrm{Np}$ 25Kh5MSGF; for continuous billet - PPAN147, PP-Np-35V9Kh3GSF; for heavy-section and rail-beam - PP-Np-25Kh5MSGF; for medium- and light-section - PP-Np-25Kh5MSGF, PP-AN147, PP-AN204; for wire - PP-Np35V9Kh3GSF, PP-AN132; for sheet - PPAN132, PP-Np-25Kh5MSGF; and for pipe ones - PP-AN147 and PP-Np-35V9Kh3GSF. However, it should be noted that the final selection of wire grade for surfacing of definite rolls is required, basing on the full-scale tests.

1. Ryabtsev, I.A., Kondratiev, I.A. (1999) Mechanized electric arc surfacing of metallurgical equipment parts. Kiev: Ekotekhnologiya.

2. Kondratiev, I.A. (1994) Self-shielded flux-cored wire for surfacing of maraging steel layer. Avtomatich. Svarka, 1, 49-51.

3. Kondratiev, I.A., Ryabtsev, I.A., Chernyak, Ya.P. (2006) Flux-cored wire for surfacing of maraging steel layer. Ibid., 4, 50-53. 